\title{
Karyotypes of Saccharomyces sensu lato species
}

\author{
Randi Føns Petersen, ${ }^{1,2}$ Torsten Nilsson-Tillgren ${ }^{2}$ and Jure Piškur ${ }^{1}$
}

Author for correspondence: Jure Piškur. Tel: +45452525 18. Fax: +45 45932809

e-mail: imjp@pop.dtu.dk

1 Department of

Microbiology, Technical

University of Denmark,

DTU-301, DK-2800 Lyngby,

Denmark

2 Department of Genetics, Institute of Molecular Biology, University of Copenhagen, Denmark

\begin{abstract}
An improved pulsed-field electrophoresis program was developed to study differently sized chromosomes within the genus Saccharomyces. The number of chromosomes in the type strains was shown to be nine in Saccharomyces castellii and Saccharomyces dairenensis, 12 in Saccharomyces servazzii and Saccharomyces unisporus, 16 in Saccharomyces exiguus and seven in Saccharomyces kluyveri. The sizes of individual chromosomes were resolved and the approximate genome sizes were determined by the addition of individual chromosomes of the karyotypes. Apparently, the genome of $S$. exiguus, which is the only Saccharomyces sensu lato yeast to contain small chromosomes, is larger than that of Saccharomyces cerevisiae. On the other hand, other species exhibited genome sizes that were 10-25\% smaller than that of $\mathbf{S}$. cerevisiae. Well-defined karyotypes represent the basis for future genome mapping and sequencing projects, as well as studies of the origin of the modern genomes.
\end{abstract}

Keywords: Saccharomyces, karyotypes, genome structure, pulsed-field electrophoresis, genome duplication

\section{INTRODUCTION}

Pulsed-field electrophoresis has made possible the separation of large yeast DNA molecules, thus allowing determination of the number and size of nuclear chromosomes, denoting the nuclear karyotype (Carle \& Olson, 1985; Johnston \& Mortimer, 1986). In addition, pulsed-field electrophoresis studies can serve as a rapid and relatively easy approach to the characterization and identification of yeast species. Yeasts belonging to the genus Saccharomyces can be divided into the sensu stricto and sensu lato groups. The sibling species of the Saccharomyces cerevisiae complex have been shown to exhibit uniform karyotypes (Cardinali \& Martini, 1994; Carle \& Olson, 1985; Naumov et al., 1992, 1995; Vaughan-Martini et al., 1993). Their chromosomes have been divided into three different classes on the basis of their size: light $(<500 \mathrm{~kb})$, medium $(500-1000 \mathrm{~kb})$ and heavy $(>1000 \mathrm{~kb})$ (Vaughan-Martini et al., 1993). Karyotype patterns of the type strains of $S$. cerevisiae and Saccharomyces paradoxus are very similar, while Saccharomyces bayanus has a species-specific karyotype (Naumov et al., 1992). Saccharomyces pastorianus (syn. Saccharomyces carlsbergensis) has been reported to be a hybrid and has been shown to possess one chromosome set originating from $S$. cerevisiae and another from a non-
S. cerevisiae parent (Nilsson-Tillgren et al., 1983; Pedersen, 1986a, b; Vaughan Martini \& Kurtzman, 1985; Vaughan-Martini \& Martini, 1987).

The karyotypes of the Saccharomyces sensu lato yeasts exhibit much heterogeneity. When these species were analysed in different laboratories, the number of bands ranged from five to 14. Saccharomyces kluyveri chromosomes may be resolved into five bands, according to Jäger \& Philippsen (1989); however, in another study, it was found to contain seven chromosomes of the heavy class that were all well above $1000 \mathrm{~kb}$ (VaughanMartini et al., 1993). In two different studies, the Saccharomyces exiguus chromosomes were separated into 11 bands (Jäger \& Philippsen, 1989) and 14 bands, of which some were double (Naumova et al., 1996). The remaining four Saccharomyces sensu lato species, Saccharomyces castellii, Saccharomyces dairenensis, Saccharomyces servazzii and Saccharomyces unisporus, were shown to contain numbers of chromosomes within these two extremes. S. unisporus and S. servazzii strains yielded chromosome banding patterns very similar to each other, exhibiting between nine and 13 bands from 580 to $2200 \mathrm{~kb}$ (Jäger \& Philippsen, 1989; Naumov et al., 1995). S. dairenensis and $S$. castellii chromosomes were resolved into eight to 11 bands with sizes from 460 to $2200 \mathrm{~kb}$ (Jäger \& 
Philippsen, 1989; Naumov et al., 1995). The results obtained in the two main studies (Vaughan-Martini et al., 1993; Naumov et al., 1995) were similar in most instances. However, until this point, chromosome identification within the Saccharomyces sensu lato yeasts was not adequate because several bands in the previous studies apparently contained overlapping chromosomes. Therefore, we focused on the development of a better separation technique, allowing the number and size of chromosomes to be determined more precisely.

\section{METHODS}

Yeast strains. The yeast species investigated in the present study were mostly type strains obtained from the Agricultural Research Service (NRRL), National Center for Agricultural Utilization Research, US Department of Agriculture, Peoria, IL, USA : $S$. cerevisiae NRRL Y-12632 ${ }^{\mathrm{T}}, S$. castellii NRRL Y-12630 ${ }^{\mathrm{T}}, S$. dairenensis NRRL Y-12639 ${ }^{\mathrm{T}}, S$. exiguus NRRL Y-12640 ${ }^{\mathrm{T}}$, S. servazzii NRRL Y-12661 ${ }^{\mathrm{T}}, S$. unisporus NRRL Y-1556 ${ }^{\mathrm{T}}$ and $S$. kluyveri NRRL Y-12651 ${ }^{\mathrm{T}}$. Non-type strains included in the study were $S$. cerevisiae YPH755, which is an ade- derivative of YPH149 (Gerring et al., 1991), S. unisporus CBS 2422 from the culture collection of the Centraalbureau voor Schimmelcultures (CBS), Delft, The Netherlands, and S. kluyveri XM 8-5 from L. Marsh, Albert Einstein College of Medicine, Bronx, NY, USA.

Preparation of chromosome-sized DNA. Chromosome plugs were prepared following a modified protocol of Gerring $e t$ al. (1991). Yeast cells were grown to stationary phase $\left(\mathrm{OD}_{600}\right.$ $10-14)$ in rich medium [YPD; 10 g yeast extract $(1 \% \mathrm{w} / \mathrm{v})$, $20 \mathrm{~g}$ peptone $(2 \% \mathrm{w} / \mathrm{v})$ and $40 \mathrm{ml} 50 \%$ glucose $(2 \% \mathrm{w} / \mathrm{v})$ $1^{-1}$. Samples $(1 \mathrm{ml})$ were spun down and washed twice in $1 \mathrm{ml}$ EDTA/Tris solution (50 mM EDTA, $10 \mathrm{mM}$ Tris, $\mathrm{pH} 7 \cdot 5$ ). Protoplasts were prepared with zymolyase $[0.15 \mathrm{ml}$ EDTA/ Tris solution plus $1 \mu \mathrm{l}$ zymolyase $\left(20 \mathrm{mg} 100 \mathrm{~T} \mathrm{ml}^{-1} 10 \mathrm{mM}\right.$ sodium phosphate buffer, $\mathrm{pH} 7 \cdot 5$ ) ] and placed at $42^{\circ} \mathrm{C}$ for 30 s. Low-melting-point agarose $(0.25 \mathrm{ml}$ of a $1 \%, \mathrm{w} / \mathrm{v}$, solution in $125 \mathrm{mM}$ EDTA, pH 7.5) was added at $42^{\circ} \mathrm{C}$ and mixed with cells. The cell suspension was gently pipetted into the plug holders and placed on ice. After the plugs had hardened they were transferred to a $12 \times 75 \mathrm{~mm}$ Falcon (glass) tube and washed as described previously (Gerring et al., 1991). Plugs were stored at $4{ }^{\circ} \mathrm{C}$ in EDTA/Tris solution. Plugs stored in this fashion are stable for several years.

Pulsed-field gel electrophoresis. Yeast chromosomes were separated by pulsed-field gel electrophoresis using either Bio-Rad CHEF-DRII or Bio-Rad Chef-Mapper. Gels were prepared as $1 \%$ ultra-pure agarose (KILOrose; Clontech) $(1.1 \mathrm{~g}$ agarose in $0.5 \times \mathrm{TBE})$. Plugs were inserted in the gel and fixed with agarose. Gels were run in $0.5 \times \mathrm{TBE}$ at $14^{\circ} \mathrm{C}$. After electrophoresis, the gels were stained in electrophoresis buffer containing $0.5 \mu \mathrm{g}$ ethidium bromide $\mathrm{ml}^{-1}$ for 4-24 h.

Separation program for Saccharomyces sensu lato chromosomes on Bio-Rad CHEF-DRII. The CHEF-DRII apparatus (Bio-Rad) allows programming of two different blocks. In order to get optimal separation of all Saccharomyces sensu lato chromosomes, a gel must be subjected to two CHEF-DRII runs. Long pulse-times allow separation of large chromosomes while shorter pulse-times give better separation of smaller chromosomes. The best separation of all chromosomes was obtained when the large chromosomes were separated first followed by separation of smaller chromosomes. The first run (Run 1) lasted for $18 \mathrm{~h}$. Run 1 was divided into block Al, pulse-time $240 \mathrm{~s}$ for $8 \mathrm{~h}$, and block B1, pulse-time $160 \mathrm{~s}$ for $10 \mathrm{~h}$. The second run (Run 2) lasted for $22 \mathrm{~h}$. Run 2 was divided into block A2, pulse-time $90 \mathrm{~s}$ for $14 \mathrm{~h}$, and block B2, pulse-time $60 \mathrm{~s}$ for $8 \mathrm{~h}$. Run 2 followed Run 1 immediately and was performed in the same buffer. It was essential to restart the CHEF-DRII between Run 1 and Run 2. The power supply was set to $150 \mathrm{~V}$.

Separation program for Saccharomyces sensu lato chromosomes on Bio-Rad Chef Mapper-multistate. The Chef Mapper-multistate program (Bio-Rad) allows for programming of several blocks with different parameters. The program used for separation of the Saccharomyces sensu lato chromosomes resembled the program designed for the CHEF-DRII. Again it was found that separation of the large chromosomes resulted in better total separation and the program was designed starting out with long pulse-times. The Chef Mapper program was divided into four blocks $(\mathrm{A}+\mathrm{B}+\mathrm{C}+\mathrm{D})$ and the total run time was $52 \mathrm{~h}$. Block A was pulse-time $240 \mathrm{~s}$ for $12 \mathrm{~h}$, block $\mathrm{B}$ was pulse-time $160 \mathrm{~s}$ for $16 \mathrm{~h}$, block $\mathrm{C}$ was pulse-time $90 \mathrm{~s}$ for $16 \mathrm{~h}$ and block D was pulse-time $60 \mathrm{~s}$ for $8 \mathrm{~h}$.

Determination of chromosome and genome sizes. The sizes of single chromosomes were calibrated against the sizes of the marker strain, S. cerevisiae YPH755. It was assumed that logarithm of the molecular mass is a linear function of the gel mobility. The total genome size was calculated by adding the sizes of single chromosomes.

\section{RESULTS}

\section{Molecular karyotypes of Saccharomyces sensu lato species}

Several different separation programs were tested on chromosomes of Saccharomyces sensu lato type strains. The results of the optimized four-step pulsed-field electrophoresis separation are shown in Fig. 1. The number and sizes of chromosomal bands together with the total sizes of the genomes were calculated on the basis of the migration of the YPH755 chromosomes. The sizes of individual chromosomes and genome sizes are depicted in Table 1.

The separation of chromosomes was not perfectly linear. Lack of linearity was particularly true for chromosomes larger than $1000 \mathrm{~kb}$. The chromosome sizes reported here are still therefore to be regarded as approximate. In addition, the intensity of single chromosomes within the same preparation varied (Fig. 1). Even though the chromosomes were separated using different programs, it is not possible to exclude completely the possibility that some single bands were actually composed of two chromosomes. This should be taken into account when the genome sizes are compared.

The four-step program generally allowed separation of all Saccharomyces sensu lato chromosomal bands. However, the resolution of bands varied slightly among different runs and different plugs and occasionally particular bands could not be distinguished. Chromosomal bands were numbered from the bottom of the gel. 
(a)
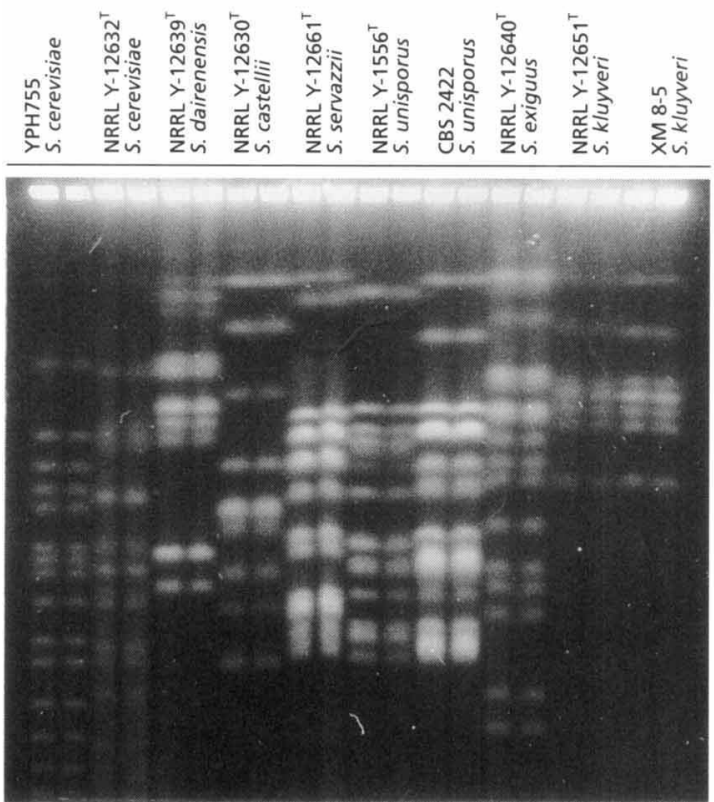

(b)

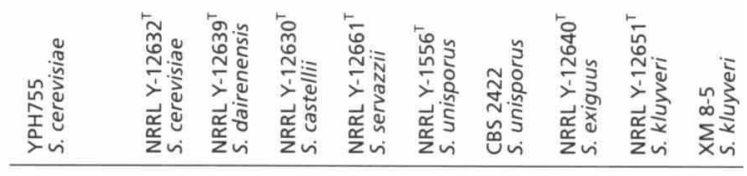

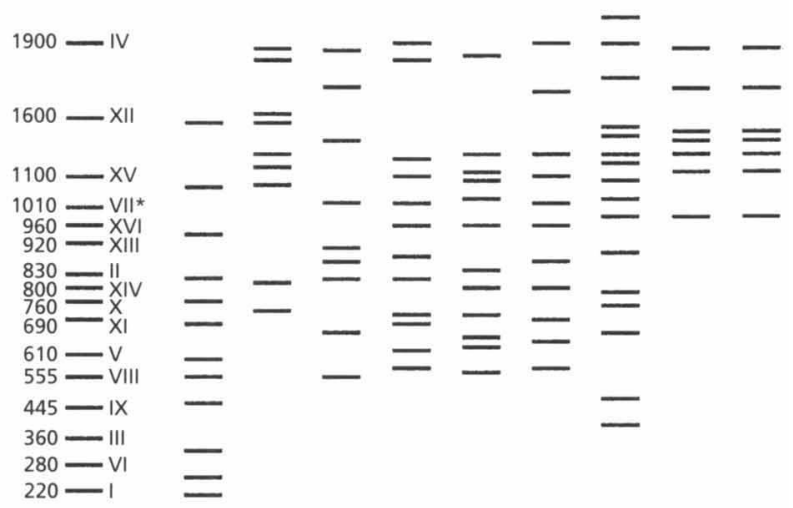

Fig. 1. Karyotypes of Saccharomyces sensu lato species. (a) Chromosome gel. (b) Schematic illustration of the separated chromosomes based on many independent experiments. Chromosome-sized DNA from S. cerevisiae YPH755, S. cerevisiae $\mathrm{Y}-12632^{\top}$, S. dairenensis $\mathrm{Y}-12639^{\top}$, S. castellii $\mathrm{Y}-12630^{\top}$, S. servazzii $\mathrm{Y}-12661^{\top}$, S. unisporus $\mathrm{Y}-1556^{\top}$, S. unisporus CBS 2422. S. exiguus $Y-12640^{\top}$, S. kluyveri $\mathrm{Y}-12651^{\top}$ and $S$. kluyveri $\mathrm{XM}$ 8-5 was separated by pulsed-field electrophoresis. The chromosomes were separated using a four-step program: step 1, pulse $240 \mathrm{~s}, 8 \mathrm{~h}$; step 2, pulse $160 \mathrm{~s}$, $10 \mathrm{~h}$; step 3, pulse $90 \mathrm{~s}, 14 \mathrm{~h}$; step 4, pulse $60 \mathrm{~s}, 8 \mathrm{~h}$. *. A YPH755 chromosome has been fragmented at RAD2 resulting in separation of chromosome VII and XV. Note also that the two $S$. unisporus strains exhibited slightly different patterns.

\section{Table 1. Numbers of chromosomes and chromosome and genome sizes of Saccharomyces sensu lato yeasts}

Chromosomes are numbered by size from the bottom of the gel (see Fig. 1). The numbers presented are in $\mathrm{kb}$ and are means, obtained by compiling the results from various pulsed-field gels. All strains are type strains except $S$. cerevisiae, which is represented by YPH149/YPH755 (Gerring et al., 1991). Note that the size of the sequenced strains of $S$. cerevisiae is approximately $13400 \mathrm{~kb}$ (Goffeau et al., 1996).

\begin{tabular}{|c|c|c|c|c|c|c|c|}
\hline & S. dairenensis & S. castellii & S. servazzii & S. unisporus & S. exiguus & S. kluyveri & S. cerevisiae \\
\hline No. of chromosomes & 9 & 9 & 12 & 12 & 16 & 7 & 16 \\
\hline \multicolumn{8}{|c|}{ Sizes of individual chromosomes: } \\
\hline 1 & 730 & 550 & 570 & 560 & 395 & 970 & 220 \\
\hline 2 & 800 & 660 & 620 & 630 & 470 & 1110 & 280 \\
\hline 3 & 1060 & 820 & 690 & 660 & 660 & 1300 & 360 \\
\hline 4 & 1200 & 860 & 720 & 720 & 740 & 1410 & 445 \\
\hline 5 & 1300 & 890 & 740 & 800 & 780 & 1510 & 555 \\
\hline 6 & 1510 & 1000 & 870 & 840 & 880 & 1750 & 610 \\
\hline 7 & 1600 & 1320 & 960 & 960 & 970 & 1950 & 690 \\
\hline 8 & 1800 & 1720 & 1010 & 1020 & 1020 & - & 760 \\
\hline 9 & 1920 & 1900 & 1100 & 1080 & 1080 & - & 800 \\
\hline 10 & - & - & 1250 & 1110 & 1250 & - & 830 \\
\hline 11 & - & - & 1850 & 1300 & 1300 & - & 920 \\
\hline 12 & - & - & 1950 & 1880 & 1470 & - & 960 \\
\hline 13 & - & - & - & - & 1510 & - & 1010 \\
\hline 14 & - & - & - & - & 1780 & - & 1100 \\
\hline 15 & - & - & - & - & 1950 & - & 1600 \\
\hline 16 & - & - & - & - & 2100 & - & 1900 \\
\hline Genome size & 11920 & 9720 & 12330 & 11560 & 18355 & 10000 & 13040 \\
\hline
\end{tabular}


Table 2. Chromosome numbers and estimated genome sizes compared with previously published results

Data were obtained from Naumov et al. (1995), Naumova et al. (1996), Jäger \& Philippsen (1989) and Vaughan-Martini et al. (1993). The sequenced strain of $S$. cerevisiae contains 16 chromosomes and its genome size, including repetitive sequences, is approximately $13400 \mathrm{~kb}$ (Goffeau et al., 1996).

\begin{tabular}{|c|c|c|c|c|c|c|}
\hline \multirow[t]{2}{*}{ Species } & \multicolumn{4}{|c|}{ Chromosome number } & \multicolumn{2}{|c|}{ Estimated genome size (kb) } \\
\hline & $\begin{array}{c}\text { Present } \\
\text { study }\end{array}$ & $\begin{array}{c}\text { Vaughan- } \\
\text { Martini }\end{array}$ & $\begin{array}{l}\text { Naumov/ } \\
\text { Naumova }\end{array}$ & $\begin{array}{c}\text { Jäger \& } \\
\text { Philippsen* }\end{array}$ & $\begin{array}{c}\text { Present } \\
\text { study }\end{array}$ & $\begin{array}{l}\text { Vaughan- } \\
\text { Martini }\end{array}$ \\
\hline S. dairenensis & 9 & 6 & 7 & 8 & 11920 & 7920 \\
\hline S. castellii & 9 & 8 & - & - & 9720 & 9415 \\
\hline S. servazzii & 12 & 9 & $9-13 \dagger$ & $10 \dagger$ & 12330 & 10430 \\
\hline S. unisporus & 12 & 11 & $9-13 \dagger$ & 10 & 11560 & 11375 \\
\hline S. exiguus & 16 & 11 & $14-16 \ddagger$ & $11 \dagger$ & 18355 & 10800 \\
\hline S. kluyveri & 7 & 7 & - & 5 & 10000 & 9550 \\
\hline
\end{tabular}

* Only a few species were represented by type strains in this study.

$\dagger$ The chromosome number for the type strain was not stated specifically.

\$Data from Naumova et al. (1996); other data in this column are from Naumov et al. (1995).

The $S$. cerevisiae type strain was included in the analysis. Only 12 bands were resolved using the fourstep program, demonstrating that a number of chromosomes ran as non-distinguishable double bands. Thus, the karyotypes obtained using pulsed-field electrophoresis may not always express the true number of chromosomes in a species. In the following analysis, our results are compared with the previously published data on Saccharomyces sensu lato type strains.

The present results show that the $S$. dairenensis type strain (Fig. 1) contains nine chromosomes with sizes ranging from 730 to $1920 \mathrm{~kb}$. The $S$. dairenensis chromosomal bands were generally well resolved and could be distinguished using the four-step program. However, bands 5 and 6 , as well as 7 and 8 , occasionally ran close to each other. The total genome size was calculated to be $11920 \mathrm{~kb}$ (Table 2), which is larger than the size reported by Vaughan-Martini et al. (1993) $(7929 \mathrm{~kb})$. Note that the number of bands observed by us in this strain was higher than that reported by Vaughan-Martini et al. (1993), Jäger \& Philippsen (1989) and Naumov et al. (1995).

S. castellii (Fig. 1) contains nine chromosomes with a size range similar to that of $S$. dairenensis. The chromosomes ranged from 550 to $1900 \mathrm{~kb}$. The $S$. castellii chromosomal bands were generally well resolved. Band 4 appeared as one band with a high intensity and may be a double band. Occasionally, band 9 was not easily detectable. It either appeared as a very weak band or it ran as a double band with band 8 . The total genome size was calculated to be $9720 \mathrm{~kb}$, which is similar to the size reported by Vaughan-Martini et al. (1993) $(9415 \mathrm{~kb})$.

Separation of S. servazzii chromosomes (Fig. 1) identified 12 chromosomal bands. Chromosome sizes ranged from 570 to $1950 \mathrm{~kb}$. With only two chromosomes belonging to the large size class, most chromosomes were medium-sized. Bands $1-4$ ran in one group; sizes ranged from 570 to $720 \mathrm{~kb}$. Bands 3 and 4 were sometimes difficult to distinguish. Band 3 was very thick. Bands 8 and 9 appeared with high intensity on the gel and may each contain two chromosomes. The genome size was calculated as $12330 \mathrm{~kb}$. Vaughan-Martini et al. (1993) reported the presence of 11 bands and a genome size of $10430 \mathrm{~kb}$ for the $S$. servazzii type strain.

As with $S$. servazzii, 12 bands were visible upon separation of chromosomes from the S. unisporus type strain (Fig. 1). Furthermore, the chromosomes were very much within the same size range, $560-1880 \mathrm{~kb}$. Bands 2 and 3 were sometimes difficult to distinguish because they often ran as a double band. Similarly, bands 9 and 10 were sometimes difficult to distinguish. The genome size was calculated to be $11560 \mathrm{~kb}$. Vaughan-Martini et al. (1993) reported 11 bands and a genome size of $11375 \mathrm{~kb}$. In the case of $S$. unisporus, two strains were analysed. They exhibited a slightly different separation pattern of nuclear chromosomes. A large chromosome of $1400 \mathrm{~kb}$ was present in CBS 2422 , but it was not present in the type strain. Instead, the type strain contained two bands of 1110 and $720 \mathrm{~kb}$. Note that the karyotypes of $S$. servazzii and $S$. unisporus exhibit a similar pattern and many chromosomes may be homologous. This is in agreement with the sequencing data on the rDNA genes which have been taken to show that these two species are closely related (James et al., 1997; Kurtzman \& Robnett, 1991; Oda et al., 1997).

Sixteen chromosomal bands were identified in $S$. exiguus (Fig. 1). The chromosome sizes ranged from 395 to $2100 \mathrm{~kb}$, thus belonging to all three size classes. Two chromosomes were smaller than $500 \mathrm{~kb}$, five 
chromosomes were between 500 and $1000 \mathrm{~kb}$ and nine chromosomes were larger than $1000 \mathrm{~kb}$. Chromosomal bands 10 and 11,12 and 13 and 15 and 16 often ran as double bands. The large number of heavy chromosomes results in the highest genome size obtained within the Saccharomyces sensu lato species, $18355 \mathrm{~kb}$. According to the present results, this species has the largest genome among Saccharomyces yeasts. Vaughan-Martini et al. (1993) reported 11 bands and a total genome size of $10800 \mathrm{~kb}$. Note that this is the only species among the Saccharomyces sensu lato species that contains small chromosomes $(<500 \mathrm{~kb})$.

S. kluyveri contained seven large chromosomes (Fig. 1). Their sizes ranged from 970 to $1950 \mathrm{~kb}$ and the genome size was $10000 \mathrm{~kb}$. All chromosomes were resolved as single bands by the four-step program. The number of chromosomes and the genome size agree with the results obtained by Vaughan-Martini et al. (1993).

The chromosome number and genome sizes obtained in the present study were compared with previously published results (Table 2). A large variation between the results from the different studies is evident. We suggest that this is due to poorer resolution obtained by the pulsed-field programs utilized in the previous studies. It may be that some chromosomes are more easily recovered upon extraction than others and that the extraction procedure is important. The discrepancy may therefore also be a result of the experimental extraction procedure (different lysing enzymes, buffers, etc.) as well as variations in the recovery of specific chromosomes.

Further discrepancies were observed in the estimated genome sizes, particularly in the case of $S$. dairenensis and $S$. exiguus. These differences are correlated with the number of chromosomes observed. In the present study, we documented a larger number of chromosomes and therefore also a larger estimated genome size for the two species. In general, $S$. kluyveri is reported to have the fewest chromosomes and to posses a genome size at the lower end of the range shown by the Saccharomyces sensu lato group. It is also interesting to point out that all species except $S$. exiguus have a smaller genome than that of the sequenced strain of $S$. cerevisiae, which is $13400 \mathrm{~kb}$ (Goffeau et al., 1996).

\section{DISCUSSION}

The genus Saccharomyces consists of several species that exhibit a lot of variation within the organization of their nuclear (Jäger \& Philippsen, 1989; Naumov et al., 1992, 1995; Naumova et al., 1996; Petersen, 1998; Vaughan-Martini et al., 1993) and mitochondrial (Groth, 1998; Petersen, 1998; Piškur et al., 1998) genomes. One possible approach to understanding the origin of the present polymorphism is to characterize and compare the organization of the modern yeast genomes. Therefore, karyotypes of type strains of some Saccharomyces sensu lato yeasts were studied in more detail. The number of chromosomes for some species belonging to the Saccharomyces sensu lato group described in this report was higher than generally reported previously (Jäger \& Philippsen, 1989; Naumov et al., 1995; Naumova et al., 1996; Vaughan-Martini et al., 1993; Table 2). In addition, the genome size estimates varied from the previous estimates (Table 2). In the case of $S$. exiguus, this difference was almost $8 \mathrm{Mb}$, and for $S$. dairenensis the difference was $3 \mathrm{Mb}$. These discrepancies are possibly due to the fact that the separation of chromosomes in our electrophoresis experiments was more efficient and thus several 'novel' distinctive chromosome bands could be detected. However, the genome sizes shown in Table 2 should still be considered as approximate for the following reasons. The lack of perfect linearity in the separation profile for chromosomes larger that $1000 \mathrm{~kb}$ may contribute to errors in the size estimates of individual chromosomes. The ploidy of several of the yeast species examined, $S$. dairenensis, $S$. servazzii, $S$. unisporus and $S$. exiguus, is not known and these yeasts are not available as monospore strains. Thus, these strains, which are likely to be diploid, may contain homologous chromosome pairs that are polymorphic and thereby produce 'extra' chromosome bands on the gel. In short, a single chromosome could be represented by two differently sized copies. On the other hand, the patterns of $S$. kluyveri and $S$. castellii type strains are the same as in the case of monospore or haploid isolates (data not shown). In addition, some chromosomes could exhibit similar electrophoretic mobility and consequently not separate into distinguishable bands. Despite these uncertainties, several conclusions can be reached from our results.

On the basis of sequence analysis of nuclear and mitochondrial genes (James et al., 1997; Kurtzman, 1993; Kurtzman \& Robnett, 1991; Oda et al., 1997; Petersen, 1998; Peterson \& Kurtzman, 1991), the Saccharomyces sensu lato yeasts can be separated into several sub-groups consisting of phylogenetically closely related species: (i) $S$. servazzii and S. unisporus, (ii) $S$. castellii and $S$. dairenensis, (iii) a group containing S. exiguus and (iv) a group containing $S$. kluyveri. It is apparent from our results (Fig. 1, Table 1) that $S$. servazzii and $S$. unisporus, two closely related yeasts, contain the same number of chromosomes and have very similar karyotypes. $S$. castellii and $S$. dairenensis, which are also relatively closely related, contain the same number of chromosomes, but only a minority of them seem to be similar in size. The numbers of chromosomes and karyotypes of $S$. exiguus and $S$. kluyveri differ substantially from any other Saccharomyces sensu lato yeasts.

The numbers and sizes of chromosomes in the Saccharomyces sensu lato group show significant heterogeneity. The Saccharomyces sensu lato chromosomes are either large or medium in size. Small chromosomes $(<500 \mathrm{~kb})$ have been detected only in S. exiguus. In other genera closely related to Saccharo- 
myces, such as Kluyveromyces, Torulaspora and Zygosaccharomyces, the chromosome number is in general lower than in $S$. cerevisiae and small chromosomes have not been detected (de Jonge et al., 1986; Oda \& Tonomura, 1995; Sor \& Fukuhara, 1989). Thus, it is likely that the progenitor of Saccharomyces and other related genera had a small number of chromosomes. However, during evolution of the genus Saccharomyces, the number of chromosomes has increased. Two different models could explain the corresponding evolutionary mechanism. One model, the 'chromosome breakage' model, suggests that some of the progenitor's large chromosomes broke and generated several smaller chromosomes. According to this model, extensive synteny should be found in the modern species. Alternatively, the 'mosaic' model proposes that the structure of the modern chromosomes is of a more recent origin and is the result of extensive gene jumping between and along chromosomes, as well as the frequent generation of novel chromosomes. According to this model, the progenitor chromosome structure cannot be deduced from the structure of the modern chromosomes. The genomes of several Saccharomyces sensu lato yeasts are currently being analysed in our laboratory to test the above models.

Another aspect of analysis of these genomes is to find out when the apparent duplication in the S. cerevisiae genome (Keogh et al., 1998; Wolfe \& Shields, 1997) took place and whether it is also present in other yeasts. Duplicated sequences in $S$. cerevisiae cover approximately $2 \mathrm{Mb}$ of the $12 \mathrm{Mb}$ sequenced genome (Wolfe \& Shields, 1997). In addition, the total genome, which is $13.4 \mathrm{Mb}$, includes approximately $1.3 \mathrm{Mb}$ of repeated sequences, such as the rDNA genes (Goffeau et al., 1996). The total genome size prior to the duplication event was suggested to be approximately 11-12 Mb (Keogh et al., 1998). It is interesting to point out that this pre-duplication size generally matches the size of a majority of the Saccharomyces sensu lato yeasts. $S$. cerevisiae and $S$. exiguus exhibit genome sizes that are larger than those of other yeasts and both contain small chromosomes (Table 2). Whether the size and number of chromosomes in $S$. exiguus are correlated with duplication of some part(s) of the genome is still not known. So far it is also unclear whether the origin of the Saccharomyces small chromosomes is mono- or polyphyletic.

\section{ACKNOWLEDGEMENTS}

We thank Thomas H. Andersen and Jeanne Hvidtfeldt for technical help and assistance and Cletus Kurtzman and Michael L. Nielsen for their comments. This work was supported by the Danish Research Council, the Carlsberg Foundation and the Novo Nordisk Foundation.

\section{REFERENCES}

Cardinali, G. \& Martini, A. (1994). Electrophoretic karyotypes of authentic strains of the sensu stricto group of the genus Saccharomyces. Int J Syst Bacteriol 44, 791-797.
Carle, G. F. \& Olson, M. V. (1985). An electrophoretic karyotype for yeast. Proc Natl Acad Sci USA 82, 3756-3760.

Gerring, S. L., Connelly, C. \& Hieter, P. (1991). Positional mapping of genes by chromosome blotting and chromosome fragmentation. Methods Enzymol 194, 57-77.

Goffeau, A., Barrell, B. G., Bussey, H. and 13 other authors (1996). Life with 6000 genes. Science 274, 546-567.

Groth, C. (1998). Saccharomyces sensu stricto yeasts: characterization of mitochondrial DNA. MSc thesis, University of Copenhagen, Copenhagen, Denmark

Jäger, D. \& Philippsen, P. (1989). Many yeast chromosomes lack the telomere-specific $\mathrm{Y}^{\prime}$ sequence. Mol Cell Biol 9, 5754-5757.

James, S. A., Cai, J., Roberts, I. N. \& Collins, M. D. (1997). A phylogenetic analysis of the genus Saccharomyces based on 18S rRNA gene sequences: description of Saccharomyces kunashirensis $\mathrm{sp}$. nov. and Saccharomyces martiniae sp. nov. Int $J$ Syst Bacteriol 47, 453-460.

Johnston, J. R. \& Mortimer, R. K. (1986). Electrophoretic karyotyping of laboratory and commercial strains of Saccharomyces and other yeasts. Int J Syst Bacteriol 36, 569-572.

de Jonge, P., de Jongh, F. C. M., Meijers, R., Steensma, H. Y. \& Scheffers, W. A. (1986). Orthogonal-field-alternation gel electrophoresis banding patterns of DNA from yeasts. Yeast 2, 193-204

Keogh, R. S., Seoighe, C. \& Wolfe, K. H. (1998). Evolution of gene order and chromosome number in Saccharomyces, Kluyveromyces and related fungi. Yeast 14, 443-457.

Kurtzman, C. P. (1993). Systematics of the ascomycetous yeasts assessed from ribosomal RNA sequence divergence. Antonie Leeuwenhoek 63, 165-174.

Kurtzman, C. P. \& Robnett, C. J. (1991). Phylogenetic relationships among species of Saccharomyces, Schizosaccharomyces, Debaryomyces and Schwanniomyces determined from partial ribosomal RNA sequences. Yeast 7, 61-72.

Naumov, G. I., Naumova, E. S., Lantto, R. A., Louis, E. J. \& Korhola, M. (1992). Genetic homology between Saccharomyces cerevisiae and its sibling species $S$. paradoxus and $S$. bayanus: electrophoretic karyotypes. Yeast 8, 599-612.

Naumov, G. I., Naumova, E. S. \& Korhola, M. (1995). Karyotypic relationships among species of Saccharomyces sensu lato: $S$. castellii, S. dairensis, S. unisporus and S. servazzii. Syst Appl Microbiol 18, 103-108.

Naumova, E. S., Naumov, G. I. \& Korhola, M. (1996). Use of molecular karyotyping for differentiation of species in the heterogeneous taxon Saccharomyces exiguus. J Gen Appl Microbiol 42, 307-314.

Nilsson-Tillgren, T., Kielland-Brandt, M. C., Holmberg, S., Petersen, J. G. L. \& Gjermansen, C. (1983). Is lager yeast a species hybrid? Utilization of intrinsic genetic variation in breeding. In Genetics of Industrial Microorganisms, pp. 143-147. Edited by Y. Ikeda \& T. Beppu. Tokyo: Kodanska.

Oda, Y. \& Tonomura, K. (1995). Electrophoretic karyotyping of the yeast genus Torulaspora. Lett Appl Microbiol 21, 190-193.

Oda, Y., Yabuki, M., Tonomura, K. \& Fukunaga, M. (1997). A phylogenetic analysis of Saccharomyces species by the sequence of $18 \mathrm{~S}-28 \mathrm{~S}$ rRNA spacer regions. Yeast 13, 1243-1250.

Pedersen, M. B. (1986a). DNA sequence polymorphisms in the genus Saccharomyces. III. Restriction endonuclease fragment patterns of chromosomal regions in brewing and other yeast strains. Carlsberg Res Commun 51, 163-183.

Pedersen, M. B. (1986b). DNA sequence polymorphisms in the 
genus Saccharomyces. IV. Homoeologous chromosomes III of Saccharomyces bayanus, S. carlsbergensis, and S. uvarum. Carlsberg Res Commun 51, 185-202.

Petersen, R. F. (1998). Genome dynamics and evolution of the mitochondrial and nuclear genomes in Saccharomyces sensu lato species. $\mathrm{PhD}$ thesis, University of Copenhagen, Copenhagen, Denmark.

Peterson, S. W. \& Kurtzman, C. P. (1991). Ribosomal RNA sequence divergence among sibling species of yeasts. Syst Appl Microbiol 14, 124-129.

Piškur, J., Smole, S., Groth, C., Petersen, R. F. \& Pedersen, M. B. (1998). Structure and genetic stability of mitochondrial genomes vary among yeasts of the genus Saccharomyces. Int J Syst Bacteriol 48, 1015-1024.
Sor, F. \& Fukuhara, H. (1989). Analysis of chromosomal DNA patterns of the genus Kluyveromyces. Yeast 5, 1-10.

Vaughan Martini, A. \& Kurtzman, C. P. (1985). Deoxyribonucleic acid relatedness among species of the genus Saccharomyces sensu stricto. Int J Syst Bacteriol 35, 508-511.

Vaughan-Martini, A. \& Martini, A. (1987). Three newly delimited species of Saccharomyces sensu stricto. Antonie Leeuwenhoek 53, 77-84.

Vaughan-Martini, A., Martini, A. \& Cardinali, G. (1993). Electrophoretic karyotyping as a taxonomic tool in the genus Saccharomyces. Antonie Leeuwenhoek 63, 145-156.

Wolfe, K. H. \& Shields, D. C. (1997). Molecular evidence for an ancient duplication of the entire yeast genome. Nature 387 , $708-713$ 